Article

\title{
Perspective of Utilization of The Spaceborne P-Band SAR Together With L/S-Band SAR
}

\author{
Boris Kutuza ${ }^{1, *}$, Anatoliy Kalinkevich ${ }^{1}$, Alexander Zakharov ${ }^{1}$, Vladimir Stasevich ${ }^{2}$, Yuri Smirnov \\ ${ }^{3}$, Vladimir Turuk ${ }^{4}$ and Victor Plushchev ${ }^{4}$ \\ 1 Kotelnikov Intstitute of Radio Engineering and Electronics, RAS, kutuza@cplire.ru, Russia \\ 2 NPP “ROBIS”, walter@robis.ru, Russia \\ 3 RSC “ENERGY”, urri-s@yandex.ru, Russia \\ 4 Corporation “VEGA”, vlturuk@gmail.com, Russia \\ * Correspondence: kutuza@cplire.ru; Tel.: +07-495-629-3365
}

\begin{abstract}
Possibilities of utilization of two frequencies synthetic aperture radar (SAR) operating simultaneously in P-band and S or L-band are considered. Advantages of such system are shown. They are caused by improvement of data interpretation and by decrease of ionosphere influence. Tasks of surface and subsurface sensing by the complex SAR were under investigation for vegetation, soil covers are given. As an example, the investigation results of coniferous forest areas backscattering by the spaceborne P-band SAR together with L-band SAR in the September-February period are presented. Studies have shown that the joint use of the results in L and P bands offer unique opportunities to explore the effects of changes in the backscattering properties of forest surfaces.
\end{abstract}

Keywords: synthetic aperture radar; P-band radar; thematic applications.

\section{Introduction}

Spaceborne P-band SAR is known to be effective instrument for the solution of topical remote sensing tasks in the area of forestry, geology, oceanology, subsurface sounding in global scale and at higher level because of better penetration of signal in P-band. The most known and effective application area of P-band SAR is a mapping of forest covers biomass. Nowadays the airborne Pband SAR systems are widely used in Russia (IMARK system), Germany, France, Sweden, etc., for the studies of natural resources. Much attention is paid to application of P-band SAR systems in studies of soil moisture, forests biomass and moisture, crops, sea ice, snow covers. The idea of P-band spaceborne SAR construction is under discussion last 15 years. One of the most known and advanced projects based on a use of P-band SAR is Biomass project, supported by ESA [1]. Similar activity is being conducted also in Russia [2]. In this experiment it is planned to place P-band SAR instrument on board of the ISS station. It should be noted that there is no SAR missions (except for SIR-C/X SAR), where simultaneous utilization of two or more SAR instruments operation in different frequency band is under consideration.

\section{P-band SAR system}

A specific feature of Russian project by now was a use of active phased array SAR antenna, but now more light-weight and worked-out hybrid mirror antenna is selected. This antenna designed by Special Design Bureau of Moscow Power Engineering Institute (SDB MPEI) was destined for S-band SAR to be placed on board of a small satellite "Condor-E" [3]. The antenna passed tests in space during ISS flight in a year 2013. In a Figure 1(a) an artist view of supposed hybrid mirror antenna on board ISS is presented. Also in the Figure 1(b) a way of the test of this antenna is presented.

\section{Technical details of P-band SAR antenna}

Hybrid mirror antenna of SDB MPEI design selected for P-band experiment is to be deployed in space. The reflector aperture shape is hexahedron with $6 \mathrm{~m}$ distance between the facets. The reflector 
focal distance is $4 \mathrm{~m}$. For dual-frequency SAR the primary feed of antenna is made in a form of three linear multi-element arrays placed in focal plane of reflector. Technical parameters of combined antenna system intended to operation in S and P-bands are as follows (see Table 1). The reflector is made of lattice structure of truss rods and joints covered with scattering surface made of knitted fabric of micro wires. The reflector weight is $63.5 \mathrm{Kg}$; the sizes are $1280 \mathrm{~mm}, 744 \mathrm{~mm}, 720 \mathrm{~mm}$. Power consumption is $30 \mathrm{~W}$.

A use of reliable hybrid antenna along with availability of ready-to-use technology of S-band SAR production lead to the idea of simultaneous exploitation the two SAR systems sharing the same antenna on board ISS station. There is a real problem of the second frequency band selection, effectively complementing P-band SAR.

Table 1: Technical parameters of antenna system

\begin{tabular}{ll} 
Frequency band (P-band), MHz & $432 \pm 30$ \\
Frequency band (P-band), MHz & $3150 \pm 100$ \\
Aperture size in P-band (vert), deg & 7.5 \\
Aperture size in P-band (vert), deg & 2 \\
Antenna gain, P-band, dB & 28 \\
Antenna gain, P-band, dB & 41 \\
Pointing, vert, P-band, deg & \pm 7 \\
Pointing, vert, S-band, deg & \pm 5 \\
Beams switching, mks & 5 \\
Polarization isolation, dB & 30 \\
Pulse power, P band, W & 500 \\
Pulse power, S-band, W & 600 \\
Average power, each band, W & 50 \\
\hline
\end{tabular}

\section{Problem of secondary band selection}

Taking in mind higher priority of forest studies, the second band should not be of too high frequency band. At the same time, higher frequencies diversity may be more fruitful from a point of view of more tasks to be solved. The images below demonstrate the potential of multi-frequency observations for remote sensing studies. In Figures 2 and 3 the images of various surface objects (forest, pit soils, villages, flood plains, etc.) are presented (images were obtained in ISTC Project No. \#2866 “Application of multi-frequency polarimetric SAR at decimeter (L,P) and meter (VHF) bands for surface and subsurface sensing of soils and vegetation covers). Numbers in a Figure 2 mark: 1 sea surfaces with various salinity, 2 - sea swell, 3 - vortex behind the rock, 4 - river mouth.

For comparison on a Figure 3 (c) is presented the radar image of forest, villages flood plains obtained by SAR "Strizh" [4,5].

When comparing $\mathrm{L}$ and $\mathrm{S}$ band signals we have to take in mind better potential resolution because of larger frequency band allocated (up to $200 \mathrm{MHz}$ ) and lower signal distortion in ionosphere in $\mathrm{S}$ band. For that reason S-band might be more attractive secondary band for P-band SAR system.

In the figures below we present for qualitative comparison a number of radar images obtained over different landscapes in various frequency bands and at different polarizations.

Preference of longer wavelengths for natural covers observation and classification is clear, but the decrease of allowable frequency bandwidth precludes discrimination of small-scale targets.

The fact that the S-band system "Strizh" mentioned above was utilizing the same reflector antenna we planned earlier for spaceborne P-band SAR to be placed on board ISS makes the idea of simultaneous use of two SAR systems to be very probable. 


\section{Interpretation of Seasonal Radar Images of Pine Forest Regions in $L$ and $P$ bands}

In [6] the results of airborne experiment on radar imaging of coniferous pine forests were presented. SAR observations were conducted over a test site with SAR system IMARC, operating in $\mathrm{X}, \mathrm{L}, \mathrm{P}$ and VHF bands. During the analysis of seasonal radar images of pine forest in $\mathrm{L}, \mathrm{P}$ and VHF bands some features with abnormal backscattering were observed. In particular, these peculiarities are most apparent in the radar images of $\mathrm{L}$ and $\mathrm{P}$ bands at $\mathrm{HH}$ polarization (Figure 4).

The comparison of two pine forest areas with different taxonomic parameters in September and November shows that backscattering from forest increases with the growth of forest biomass. Although in February the difference in reflective properties of selected regions disappears. At the same time in $\mathrm{P}$ band the opposite behaviour of reflective properties is observed: in February the difference of backscattering of selected areas grows sharply. In autumn the difference in scattering properties of two regions in $\mathrm{P}$ band is weak. It should be noted that radar imaging in February was carried out under cold weather conditions - in certain days the surface temperature reached $-25^{\circ} \mathrm{C}$. One can consider that taxonomic parameters of selected pine forest areas did not change from September to February. The regions are located near each other, characteristics of the surface in these areas should vary in similar way, what is proved by the results of summer ground-based measurements of soil content and subsurface water level, seasonal properties of wood for both pine forest regions should also be similar. In accordance with this the observed behaviour of reflective characteristics of pine forest regions should be interpreted accordingly. It was assumed that peculiar properties of radar backscattering of the forest in $\mathrm{L}$ and $\mathrm{P}$ bands may be explained by the seasonal variation of water status of individual tree elements (trunks, branches and needles).

\subsection{Absorption and Scattering in the Forest}

The architecture of pine forest is complicated. As a first approximation forest may be represented as two-layered medium consisting of crown layer (needles, branches and trunks) layer, where crown layer is at the top. In the case of observations in short wavelengths band the crown is an effective element of scattering and mainly defines the effect of backscatter saturation on biomass. Tree trunks are the largest elements of forest cover. Thus one can expect that the signal will be scattered by trunks more intensively. At the same time it is necessary to take into account the role of crown in absorption and scattering of waves. In high-frequency range the role of crown is dominant in backscattering. Thereby the total frequency dependence of backscattering is governed by the counteraction of two factors: the increase of trunks cross-section at higher frequencies and simultaneous growth of absorption of waves in tree crown and increasing of backscattering cross-section of conifer elements. As a result, one can expect the presence of maximum and minimum in frequency dependence of total scattering cross-section.

The absorption and scattering of waves are directly related with dielectric properties of tree elements. Thus it was suggested to relate peculiarities of reflective characteristics firstly with seasonal variations of geophysical parameters of tree elements. Dielectric permittivity of vegetation elements such as trunks, branches, needles and other is defined by a composition of solid cellulose and water solution. In [7] an expression is given for complex dielectric permittivity of tree elements depending on moisture, temperature, frequency of radiation. The analysis of this expression shows that real and imaginary parts of dielectric permittivity are rather high even at comparatively low volume moisture and strongly depend on it. It was assumed that abnormal behaviour in reflective properties is related with specific moisture variation of individual elements of pine tree (needles, branches and trunks).

In 50 s the intensive study of water regime of various sorts of wood vegetation was performed. More than $50 \%$ of total wet tree mass consists of water. The water content varies widely in different parts of a tree and depends on tree type, its age, place of growth and season. Some types of wood vegetation experience showed strong seasonal variations of water content in trunks. Although even for similar types of vegetation seasonal variation of moisture may be different. For example, Gibbs has discovered rather weak variation for conifers in East Canada, but at the same time he addresses the data of R. Gartig and A. Munch, who observed considerable seasonal variation of water content 
in conifers [8]. Thus, there is no strict law of seasonal water content variation and data relating weather conditions particular in winter with water state of conifers is missing.

\subsection{Results of Ground-Based Measurements of Tree Water Status}

In order to explain seasonal variation of water status of pine forests in seven test sites from September 2008 to July 2009 the measurements of taxonomic tree parameters were conducted as well as of seasonal variations of weight moisture of trunks, needles and branches. Selected test sites have various taxonomic parameters. Special attention was given to the sites located in close vicinity to each other and having peculiar reflectivity properties described above. Significant attention was also paid to weather conditions, when taking wood samples the temperature conditions identical to the conditions of experiment were chosen.

It is known that moisture of trunk varies in height and depth, depends on the place of tree growth, etc. Thus value of moisture may strongly fluctuate from one tree to another. It was decided to conduct seasonal measurements of moisture using several groups of trees in each test site and to compare obtained dependencies. As a measure of trunk moisture the wood samples taken at a height of $130 \mathrm{~cm}$ from ground surface and from depths of 0-6 cm were taken. The disadvantage of this technique was necessity to take numerous samples from the same tree trunks. We selected trees whose needles and branches were available for sampling (typically the tree crown was $15 \mathrm{~m}$ higher than ground surface). The sampling was carried out from 11 a.m. to 01 p.m. of local time. In Figure 5 the dependencies of water content variation in trunks (a), branches (with diameter less than $15 \mathrm{~mm}$ ) (b) and needles (c) are given. The lines represent the results of measurements for single trees from the test sites with different reflective properties. One can clearly trace the identical character of moisture variation over seasons. The points represent measurement results for other trees. Similarly, in Figure 5(b) the dependencies of moisture content variation in branches are given. Weight moisture content of branches and needles varies strongly but needle dependence is very close to the dependence of branches.

Obtained dependencies show that seasonal character of trunk wood moisture content differs from those of thin branches and needles. Considerable decrease of branch moisture in cold time fell the beginning of increase of trunk moisture content.

Analysing the dynamics of seasonal variation of tree elements the following conclusions may be drawn:

from September to July moisture content of trunks, branches and needles varies considerably particularly in cold season;

for each single tree or for the forest in general a clear dependence of wood moisture content on time may be traced;

annual plots of moisture content of needles and branches are identical;

in cold season the behaviour of moisture variation in trunks and in branches differs considerably.

\subsection{The modelling of Radar Backscatter from Trees}

When modelling the branches, needles and trunks were represented as dielectric cylinders. The notion of "thin" cylinder refers to a case when wavelength inside the cylinder is much longer than cylinder radius. Otherwise cylinders should be considered thick. The transition point between thin and thick cylinders is defined by a condition

$$
\frac{2 \pi a f}{c} \sqrt{|\varepsilon|}=1
$$

Here $a$ is cylinder radius, $f$ is radar frequency, $c=3 \cdot 10^{10}$ is the speed of light. Based on this equation one can define the transition frequency between calculations in approximations of thin and thick cylinders. For the case of $a=0.35 \mathrm{~cm}$ and $m_{v}=0.5$ transition frequency is approximately $2 \mathrm{GHz}$. Taking into account that dielectric permittivity varies considerably with moisture variations, one can see that in one season single elements of conifer tree may be regarded as thin dielectric cylinders while in 
other season as thick. Tree trunks for L and P band waves may be considered as a layer of vertical thick cylinders, needles as a layer consisting of thin cylinders independently on weather conditions. For branches the situation is different. They should be considered as thin dielectric cylinders in certain periods and as thick cylinders in other time.

For estimation of backscattering two terms should be taken into account: differential scattering cross-section $\sigma d$ and total cross-section $\sigma$. First one is needed for the determination of backscattering by forest elements while the other is used for calculation of waves' attenuation.

For the case of thin cylinder backscattering cross-section depends strongly on the polarization of incident wave. For vertical polarization the scattering cross-section is approximately a cone. It means that scattering intensity is defined only by angle between cylinder axis and direction of scattering. Relevant scattering cross-section is described by a formula

$$
\sigma_{d}=\frac{|\varepsilon-1|^{2}(k a)^{4} l^{2} \sin ^{2} \vartheta_{i} \sin ^{2} \theta}{16} \operatorname{sinc}^{2}\left[\frac{k l}{2}\left(\cos \vartheta_{i}-\cos \theta\right)\right]
$$

$k=2 \pi f / c$ is wavenumber, $l$ is cylinder length, $\theta_{i}$ is angle between incidence direction and cylinder axis, $\theta$ is angle between cylinder axis and scattering direction, $\operatorname{sinc} x=\sin x / x$. For large values of $k l$ product backscattering is concentrated near "specular" direction defined by the equation $\cos \theta=\cos$ $\theta_{i}$. (it is important when considering reflection from trunks towards ground and reflection from ground towards radar).

If incident wave electric field direction is orthogonal to the cylinder axis, the cross-section for this polarization is $4 /|\varepsilon+1|^{2}$ times less then when the electric field is directed along the cylinder axis. Taking into account that $\varepsilon>1$, scattering cross-section at horizontal polarization is less than at vertical one.

Scattering cross-section of thick cylinder in the directions lying in the incidence plane is given by expression [9]

$$
\sigma_{d}^{\leftarrow}=\frac{k a l^{2}}{4 \pi} \sin \vartheta_{i} \operatorname{sinc}^{2}\left[\frac{k l}{2}\left(\cos \vartheta_{i}-\cos \theta\right)\right] F^{2}\left(\pi / 2-\vartheta_{i}\right)
$$

Here $F=F_{H}(\beta)=\frac{\cos \beta-\sqrt{\varepsilon-\sin ^{2} \beta}}{\cos \beta+\sqrt{\varepsilon-\sin ^{2} \beta}}$ is Fresnel coefficient for horizontally polarized waves and $F=F_{V}(\beta)=\frac{\varepsilon \cos \beta-\sqrt{\varepsilon-\sin ^{2} \beta}}{\varepsilon \cos \beta+\sqrt{\varepsilon-\sin ^{2} \beta}}$ is Fresnel coefficient for vertically polarized waves.

Total scattering for thin cylinder in case of vertical polarization is given by formula

$$
\sigma_{t}=\varepsilon^{\prime \prime} k l \pi a^{2} \sin ^{2} \vartheta_{i}
$$

where $\varepsilon^{\prime \prime}$ is imaginary part of dielectric permittivity, $v=\pi a^{2} l$ is cylinder volume. In case of thick cylinder cross-section is independent of polarization and equals to:

$$
\sigma_{t}=4 a l \sin ^{2} \vartheta_{i} .
$$

When estimating total cross-section of needles it is assumed that their orientation is arbitrary i.e. $\sin ^{2} \theta_{i}$ should be substituted by its mean value equal to $1 / 2$. Thus for attenuation factor of waves by needles the relation $\gamma_{n}=\frac{1}{2} \varepsilon " k v N$ may be used, where $N$ is concentration of needles in a unit of volume. With decrease of temperature, when moisture is decreasing also, the attenuation in needles decreases. For spherical frame with axis $z$ along the tree trunk azimuth direction of branches $\varphi$ may be considered uniform within the limits $[0,2 \pi]$ and probability distribution of angle $\theta$ between axis $z$ and tree branch may be specified by some function. For estimations the following function was used:

$$
P_{1}\left(\vartheta_{g}\right)=\frac{32}{3 \pi} \sin ^{4} 4 \vartheta_{g}, \quad 0 \leq \vartheta_{g} \leq \frac{\pi}{4} .
$$


For estimations we used the results of work [8], where parameters of single elements of pine forest depending on their age were given. In calculations we supposed the soil to be sandy one with $50 \%$ moisture.

Given assumptions provided basis for rough estimates of reflective properties of forest cover.

Preliminary calculations show that specific effective scattering cross-section (with regard of needles, branches and trunks) of conifer tree has complex character (Figure 6). In these dependencies maximum and minimum values are observed, which are caused by a presence of two factors: increase of cross-section with frequency and simultaneous increase of attenuation by tree crown and increase of of needles and branches cross-section. Values of maximums and minimums and their location in frequency axis are substantially defined by dielectric properties of tree elements.

Obtained experimental data on tree moisture and results of modelling calculations allow us to make an attempt to explain observed seasonal peculiarities of radar reflectivity of pine forest in $L$ and $P$ bands. Direct dependence of reflectivity on biomass in L band in warm time (SeptemberNovember) is related with relatively high concentration of water in tree crown and with the influence of attenuation and volume scattering by branches. At the same time for $\mathrm{P}$ band the weak dependence of scattering on moisture may be explained by higher attenuation in crown. For cold time when crown moisture decreases sharply, attenuation in needles in L band may be neglected and backscattering is defined by sufficiently dry crown branches. Reflectivity of pine forest in P band at low temperatures is related with reflection from trunks.

\section{Expected Results}

An international experience of spaceborne SARs exploitation shows that significant amount of remote sensing tasks might be solved more efficiently using SAR data obtained in various frequency bands because of different penetrating capability of signals into sounding media and sensitivity to surface roughness. Joint utilization of $\mathrm{S}$ and $\mathrm{P}$ band data is an attractive combination because of essential difference of the signals interaction with sounding media as well as with propagation wave in ionosphere.

Remote sensing tasks frequently propose underlying covers sounding with subsurface penetration. $\mathrm{P}$ and $\mathrm{S}$ band data are preferable for vegetation studies. Because of distinction in signals penetration depths it is possible to study height profile of forest, to study forest biomass in more detail and to measure thickness of vegetation layer by means of interferometry technique.

In the area of vegetation covers studies such as forests it is possible to conduct forests classification more efficiently and to measure vegetation biomass because of better penetration of $\mathrm{P}$ band signals under the tree crown and sensitivity to signal backscatter from large branches and trunks. At the same time, it is possible to study soil state under the forest covers, moisture extent, detection of forest floods, while $\mathrm{S}$ band signals provide sensitivity to penetration from crowns and smaller branches.

An estimation of soils state in economic activity, such as moisture, it is maybe evaluated more efficiently in P-band because of lower influence of vegetation and higher penetration depth in subsurface sounding, though the landscapes classification according to their small-scale roughness (at wavelength scale) is preferable in S-band, where almost all the surface types are rough ещin different extent.

In geology it is possible to observe geological structures under the sediments and vegetation in $\mathrm{P}$ band. For the same reason this frequency band is preferable for studies of arid and desert areas.

In hydrology longer wavelengths are preferable in studies of wet underlying surface layers because of lower influence of surface roughness and sensitivity щт larger depth of surface layer. Water reservoirs may be delineated more reliably because of lower influence of wind ripples on the water surface. At the same time, the maps of large-scale waves separated from wind ripples are observable better in P-band. Studies of flood zones covered with forests are more efficient in P-band because of better radio waves penetration through the forest layer.

In glaciology P-band provides better opportunities for studies of structure of ice covers of shallow fresh-ice water reservoirs because of better signal penetration through the ice layer and 
interaction with bottom topography, yet S-band data are preferable for sea ice covers classification because of coincidence of wave length and scales of ice roughness. Studies of ice glaciers and glaciers structures under the snow covers as well as permafrost soils are preferable in P-band also.

In the interferometric scheme of surface mapping from satellite repeated orbits the P-band provides lower sensitivity of measurements to temporal decorrelation of backscattered signals, especially in the case of long time intervals between observations, when observations at shorter wavelengths are practically impossible.

In studies of the atmosphere impact of the use of $S$ and $P$ bands will allow studies of destructive influence of ionosphere on the process of radar images synthesis and search for algorithms of compensation of the radar images defocusing, Faraday rotation of linearly polarized signal polarization plane orientation as well as unwanted phase shifts corrupting interferometric measurements of surface topography.

Author Contributions: Boris Kutuza and Anatoliy Kalinkevich proposed the idea of experiment and the paper structure; Alexander Zakharov contributed in analysis of SAR applications; Vladimir Turuk, Vladimir Stasevich, Victor Pluschev and Yuri Smirnov contributed technical description of SAR system and ISS onboard technical capabilities; Anatoliy Kalinkevich wrote a paper.

Conflicts of Interest: The authors declare no conflict of interest. The founding sponsors had no role in the design of the study; in the collection, analyses, or interpretation of data; in the writing of the manuscript, and in the decision to publish the results. 


\section{References}

1. T. Le Toan, at al. "The BIOMASS Mission: Mapping global forest biomass to better understand the terrestrial carbon cycle, Remote Sensing of Environment, 2010.

2. B.Kutuza, A.Kalinkevich, A.Zakharov, V.Stasevich, Yu.Smirnov, Space experiment on the International Space Station with P-band SAR// Proceedings of EUSAR-2014 Conference, Berlin, Germany, 02-06 June, 2014.

3. L.Neronsky, I.Osipov, V.Turuk, V.Andrianov, I.Porokhov, Choice of Engineering Performances in Designing Multipurpose Synthetic Aperture Radar for Small Satellites// Proceedings of EUSAR 2002 Conference, Cologne, Germany, 04-06 June, 2002.

4. Verba V.S., L.B.Neronsky, L.G.Osipov, V.E.Turuk Spaceborne Earth Surveillance Radar Systems, M.Radiotechnika, 2010 (in Russian) English edition 2016 (in press).

5. Turuk V.E., Verba V.S., Golovanova M.V., Golubtzov P.E., Kurenkov V.N. Neronskiy L.B., Zaitzev S.E., Tolstov E.F. Preliminary results of flight testing the SAR "Strizh" as part of Light Satellite "Condor-E" type // Proceedings of EUSAR'2016; Hamburg Germany, June 06-09, 2016.

6. Kalinkevich A.A., Manakov V.Yu., Armand N.A., Krylova M.S., On the mechanism of EV waves backscattering by pine forest in meter band. Radiotehnika i Elektronika, vol. 53, no.10, 2008

7. Ulaby F.T., Sarabandi K., McDonald K., et al, Michigan microwave canopy scattering model, Int. J. Remote Sensing, 1990, v. 11, No 7, pp 1223 - 1253.

8. Hsu C.C., Han H.C., Shin R.T. et al, Radiative transfer theory for polarimetric remote sensing of pine forest at P band, Int. J. Remote Sensing, 1994, v. 15, No 14, pp $2943-2954$.

9. N.A. Armand, V.M. Polyakov, Radio Propagation and Remote Sensing of the Environment, CRC PRESS, London New York Washington D.C.2005. 